\title{
Additive Angle Method for Fast Large-Tip-Angle RF Pulse Design in Parallel Excitation
}

\author{
William A. Grissom, ${ }^{1 *}$ Chun-Yu Yip,${ }^{2}$ Steven M. Wright, ${ }^{3}$ Jeffrey A. Fessler, ${ }^{1,2}$ \\ and Douglas C. Noll ${ }^{1}$
}

\begin{abstract}
Current methods for parallel excitation RF pulse design are based on the small-tip-angle approximation, which provides a computationally efficient means of pulse calculation. In general, pulses designed with those methods are inaccurate when scaled to produce large-tip angles, and methods for large-tipangle pulse design are more computationally demanding. This paper introduces a fast iterative method for large-tip-angle parallel pulse design that is formulated as a small number of Bloch equation simulations and fast small-tip-angle pulse designs, the results of which add to produce large-tip-angle pulses. Simulations and a phantom experiment demonstrate that the method is effective in designing multidimensional large-tip-angle pulses of high excitation accuracy, compared to pulses designed with small-tip-angle methods. Magn Reson Med 59:779-787, 2008. (C) 2008 Wiley-Liss, Inc.
\end{abstract}

Key words: pulse design; parallel excitation; transmit SENSE; multidimensional pulse; large-tip-angle pulse

\section{INTRODUCTION}

$\mathrm{RF}$ pulses designed for accelerated parallel excitation using small-tip-angle methods (1-4) produce inaccurate excitation patterns when scaled to large-tip-angles (4). Although unaccelerated pulses designed with small-tip-angle (5) methods can produce accurate large-tip-angle excitation patterns if they satisfy the "linear-class" conditions $(6,7)$, at high acceleration factors, increased pulse magnitudes violate the small-RF approximation used to derive the linear class. Several large-tip-angle pulse design methods have recently been proposed specifically for parallel excitation (8-10). These methods either suffer from long computation times, or achieve shorter computation times via pulse parameterization, which limits the degrees of freedom in pulse design, and locks the designer into using certain classes of excitation k-space trajectories. The computational bottleneck of most large-tip-angle methods is the Bloch equation, which must be evaluated repeatedly to obtain the magnetization profile subsequent to excitation, and in some methods to calculate the gradient of a cost function with respect to RF pulse samples. In this work,

\footnotetext{
${ }^{1}$ Biomedical Engineering, University of Michigan, Ann Arbor, Michigan

${ }^{2}$ Electrical Engineering and Computer Science, University of Michigan, Ann Arbor, Michigan

${ }^{3}$ Electrical and Computer Engineering, Texas A\&M University, College Station, Texas

Grant sponsor: NIH; Grant number: DA15410

*Correspondence to: William Grissom; 2360 Bonisteel Ave, Ann Arbor, MI 48109-2108, USA. E-mail: wgrissom@umich.edu

Received 23 June 2007; revised 9 October 2007; accepted 20 November 2007. DOI 10.1002/mrm.21510

Published online in Wiley InterScience (www.interscience.wiley.com).

(c) 2008 Wiley-Liss, Inc.
}

we propose a large-tip-angle pulse design method that requires only a small number of Bloch equation simulations, significantly reducing computation time compared to other methods. Furthermore, our method is formulated as a series of small-tip-angle pulse designs that can be performed rapidly using nonuniform fast Fourier transforms (NUFFT's) (11). Our method is also simple to implement, using existing software for designing small-tip-angle parallel RF pulses.

The method we propose is based on a series of iterative updates to a large-tip-angle pulse. Initially, a pulse is designed using a linear small-tip-angle method. This pulse is then simulated using a Bloch equation simulator, and we expect that the flip angle pattern excited by the pulse will deviate significantly from the desired flip angle pattern. A small-tip-angle pulse is then designed to add to the first pulse, to bring the pattern excited by the summed pulses closer to the desired pattern. The summed pulse is seeded to the next iteration, and iterations continue until a convergence criterion is met. We show in simulations and experiments that this method produces $\frac{\pi}{2}$ - and $\pi$-excitation pulses with significantly improved accuracy over pulses designed using small-tip-angle methods, for both spiral and echo-planar (EP) trajectories.

One-dimensional large-tip-angle RF pulse design methods have been proposed that are similar to the one we present here $(12,13)$. Ref. 13 proposes an iterative scheme to design large-tip-angle pulses. At each step in the design, the current pulse is Bloch-simulated, and a pulse is designed that adds to the current pulse, to bring the excited pattern closer to the desired one. In (13), the pulses are constrained to be real and time symmetric, to ensure that they all rotate magnetization about the same vector everywhere in space, and as a result their flip angles sum coherently. Our approach differs from this method in that we allow pulses to possess time-varying phase, so the vectors about which the pulses rotate magnetization can vary both spatially and between design iterations, and we employ the Fourier small-tip-angle approximation to accelerate pulse computation. Ref. 12 proposes an iterative scheme to refine small-tip-angle pulses whose excited patterns are corrupted by scanner imperfections. In that method, pulses are designed using Fourier analysis. Our approach is perhaps most similar to that method, however, we introduce a modification that allows us to design accurate large-tip-angle pulses. Additionally, to our knowledge, this work also represents the first application of this class of pulse design methods to multidimensional pulse design for parallel excitation. 


\section{THEORY}

Consider parallel excitation pulse design for $R$ coils. Assume we are given $N_{S}$ samples of a desired tip angle pattern $\theta^{\text {des }}(\mathbf{x})$ and an initial set of pulses $\left\{\mathbf{b}_{1}, \ldots, \mathbf{b}_{R}\right\}$, where $\mathbf{b}_{r}$ is a length- $N_{t}$ vector of pulse samples for the $r$ th coil. If $\left\{\mathbf{b}_{1}, \ldots, \mathbf{b}_{R}\right\}$ are small-tip-angle pulses, the tip angle pattern they excite at spatial position $\mathbf{x}_{i}$ is well approximated by $(4,6)$ :

$$
\begin{array}{r}
\theta\left(\mathbf{x}_{i}\right) e^{i \angle M_{x y}\left(\mathbf{x}_{i}\right)} \approx i \gamma \Delta t \sum_{r=1}^{R} s_{r}\left(\mathbf{x}_{i}\right) \sum_{j=1}^{N_{t}} b_{r, j} e^{i \mathbf{x}_{i} \cdot \mathbf{k}\left(t_{j}\right)}, \\
i=1, \ldots, N_{s},
\end{array}
$$

where $\theta(\mathbf{x})$ is the tip angle excited by the pulses, $\angle M_{x y}(\mathbf{x})$ is the phase of the resulting transverse magnetization, $s_{r}(\mathbf{x})$ is coil r's transmit sensitivity pattern, and $\mathbf{k}(t)$ is the excitation k-space trajectory defined by reverse integration of the gradient waveforms (5). Further assume that $\left\{\mathbf{b}_{1}, \ldots, \mathbf{b}_{R}\right\}$ do not excite $\theta^{\text {des }}(\mathbf{x})$ accurately, and that the difference between the desired and excited patterns, $\theta^{\text {new }}(\mathbf{x}) \triangleq$ $\theta^{\text {des }}(\mathbf{x})-\theta(\mathbf{x})$, is a small-tip-angle pattern. Our goal is to design a new set of pulses $\left\{\tilde{\mathbf{b}}_{1}, \ldots, \tilde{\mathbf{b}}_{R}\right\}$ that, when added to the initial pulse set, results in a set that excites $\theta^{\text {des }}(\mathbf{x})$. To achieve this, we design $\left\{\tilde{\mathbf{b}}_{1}, \ldots, \tilde{\mathbf{b}}_{R}\right\}$ to satisfy:

$$
\begin{array}{r}
\theta^{\text {new }}\left(\mathbf{x}_{i}\right) e^{i \angle M_{x y}\left(\mathbf{x}_{i}\right)} \approx i \gamma \Delta t \sum_{r=1}^{R} s_{r}\left(\mathbf{x}_{i}\right) \sum_{j=1}^{N_{t}} \tilde{b}_{r, j} e^{i \mathbf{x}_{i} \cdot \mathbf{k}\left(t_{j}\right)}, \\
i=1, \ldots, N_{s},
\end{array}
$$

where $\angle M_{x y}(\mathbf{x})$ is obtained from Eq. [1], i.e., it is the transverse phase induced by the initial pulses. Setting $d^{\text {new }}(\mathbf{x}) \triangleq \theta^{\text {new }}(\mathbf{x}) e^{i \angle M_{x y}(\mathbf{x})}$, we can design $\left\{\tilde{\mathbf{b}}_{1}, \ldots, \tilde{\mathbf{b}}_{R}\right\}$ using the iterative small-tip-angle parallel pulse design method of (4), which will calculate pulses that minimize the cost function:

$$
\Psi\left(\tilde{\mathbf{b}}_{1}, \ldots, \tilde{\mathbf{b}}_{R}\right)=\left\|\sum_{r=1}^{R} \mathbf{S}_{r} \mathbf{A} \tilde{\mathbf{b}}_{r}-\mathbf{d}^{\mathrm{new}}\right\|_{\mathbf{W}}^{2}+\beta \sum_{r=1}^{R}\left\|\mathbf{b}_{r}+\tilde{\mathbf{b}}_{r}\right\|^{2},
$$

where $\mathbf{S}_{r}$ is a diagonal matrix containing samples of $s_{r}(\mathbf{x})$ and $\mathbf{A}$ is a nonuniform fast Fourier transform (NUFFT) matrix corresponding to $e^{i \mathbf{x}_{i} \cdot \mathbf{k}\left(t_{j}\right)}(14)$. The term $\sum_{r=1}^{R} \mathbf{S}_{r} \mathbf{A} \tilde{\mathbf{b}}_{r}$ is a matrix/vector expression for the right-hand side of Eq. [2]. $\mathbf{W}$ is a diagonal matrix containing a spatial error weighting that can be used to specify a region of interest (ROI) (4). Integrated power of the total RF pulse may be controlled via the Tikhonov regularization term $\beta \sum_{r=1}^{R}\left\|\mathbf{b}_{r}+\tilde{\mathbf{b}}_{r}\right\|^{2}$, where $\beta$ is a tuning parameter. We minimize $\Psi\left(\tilde{\mathbf{b}}_{1}, \ldots, \tilde{\mathbf{b}}_{R}\right)$ using the Conjugate Gradient (CG) method. Because of linearity of excitation in the small-tipangle regime, and because the tip angle patterns excited by the new and initial pulses are in phase, $\left\{\mathbf{b}_{1}+\tilde{\mathbf{b}}_{1}, \ldots, \mathbf{b}_{R}+\right.$ $\left.\tilde{\mathbf{b}}_{R}\right\}$ will accurately excite the tip angle pattern $\theta^{\text {des }}(\mathbf{x})$. In short, we are given an initial pulse set that excites an erroneous tip angle pattern, and we design a set of correction pulses that excite the difference in tip angle between the erroneous and desired patterns. When the initial and correction pulses are summed, the result is a pulse set that excites an accurate pattern, in the small-tip regime.

Now consider the more challenging pulse design scenario in which $\theta^{\text {des }}(\mathbf{x})$ is a large-tip-angle desired pattern, and $\left\{\mathbf{b}_{1}, \ldots, \mathbf{b}_{R}\right\}$ are inaccurate initial large-tip-angle pulses. Equation [1] no longer holds in this case, and we must use Bloch equation simulation to obtain $\theta(\mathbf{x}) e^{i \angle M_{x y}(\mathbf{x})}$, where $\theta(\mathbf{x})=\cos ^{-1} \frac{M_{z}(\mathbf{x})}{M_{0}(\mathbf{x})}$. Furthermore, for larger tipangles the right-hand side of Eq. [2] becomes an increasingly inaccurate model for the difference between the tip angles excited by $\left\{\mathbf{b}_{1}, \ldots, \mathbf{b}_{R}\right\}$ and $\left\{\mathbf{b}_{1}+\tilde{\mathbf{b}}_{1}, \ldots, \mathbf{b}_{R}+\tilde{\mathbf{b}}_{R}\right\}$. Despite this, we have found empirically that if the initial pulses $\left\{\mathbf{b}_{1}, \ldots, \mathbf{b}_{R}\right\}$ excite angles smaller than $180^{\circ}$, then the pulses $\left\{\mathbf{b}_{1}+\tilde{\mathbf{b}}_{1}, \ldots, \mathbf{b}_{R}+\tilde{\mathbf{b}}_{R}\right\}$ will still excite a more accurate pattern. That is, while the linear model of Eq. [2] and the true difference in flip-angle patterns are significantly different when the initial pulses excite large-tip angles, the difference is not so great as to prevent an improvement in accuracy when $\left\{\tilde{\mathbf{b}}_{1}, \ldots, \tilde{\mathbf{b}}_{R}\right\}$ are designed using Eq. [2]. In contrast to the linear smalltip-angle case, the desired level of accuracy generally cannot be reached with only one design of $\left\{\tilde{\mathbf{b}}_{1}, \ldots, \tilde{\mathbf{b}}_{R}\right\}$. To make further improvements, we adopt a fixed-point iterative procedure to update the initial pulses multiple times, until accuracy stops improving. It is usually necessary to incorporate the transverse magnetization phase term, $e^{i \angle M_{x y}(\mathbf{x})}$, into $d^{\text {new }}$ so that the flip angle pattern produced by $\left\{\tilde{\mathbf{b}}_{1}, \ldots, \tilde{\mathbf{b}}_{R}\right\}$ adds with the intended sign. If the phase term is not incorporated, the algorithm may stop improving excitation accuracy before an acceptable error level is reached.

In summary, the additive angle pulse design method is an iterative technique that improves the accuracy of a large-tip-angle RF pulse. At each iteration, a Bloch equation simulation is performed to obtain $\theta(\mathbf{x}) e^{i \angle M_{x y}(\mathbf{x})}$ for the initial pulses, and new pulses are designed to excite $\theta^{\text {new }}(\mathbf{x}) e^{i \angle M_{x y}(\mathbf{x})}$ that are added to the initial pulses, where $\theta^{\text {new }}(\mathbf{x}) \triangleq \theta^{\text {des }}(\mathbf{x})-\theta(\mathbf{x})$. The summed pulses then replace the initial pulses in the next iteration. Iterations continue until excitation accuracy ceases to improve.

\section{METHODS}

\section{Pulse Design}

We used the additive angle method to design twodimensional RF pulses, and compared it to small-tip-angle $\mathrm{RF}$ pulse design in simulations and experiments. Desired patterns were specified on a $64 \times 64$ grid, with a FOV of $24 \mathrm{~cm} \times 24 \mathrm{~cm}$, and were blurred by convolution with a Gaussian kernel of FWHM = $1.2 \mathrm{~cm}$, to reduce ringing in the resulting excitation patterns. Bloch equation simulations for pulse design were performed on the same grid, while simulations for final error computation and comparison were performed on a finer $128 \times 128$ grid with the same FOV. Bloch simulations for pulse design were initialized with all magnetization in $M_{z}$. Spins outside the ROI were not simulated. 50 CG iterations were used for each small-tip-angle pulse design. To ensure that $\left\{\tilde{\mathbf{b}}_{1}, \ldots, \tilde{\mathbf{b}}_{R}\right\}$ 
stay in the small-tip-angle regime, we scaled $d^{\text {new }}(\mathbf{x})$ to a maximum tip angle of $\frac{\pi}{4}$ radians, except in simulation II, where the maximum tip angle was varied. We stopped the additive-angle iterations when the Root-mean-square error (RMSE), defined as RMSE $=\frac{1}{N_{s}} \sqrt{\sum_{i=1}^{N_{s}}\left|\theta^{\operatorname{des}}\left(\mathbf{x}_{i}\right)-\theta\left(\mathbf{x}_{i}\right)\right|^{2}}$, between the desired and Bloch-simulated tip-angle patterns at the current iteration decreased by less than $0.01 \%$ of the previous one. For conventional small-tip design, we used the method in (4), with the same desired flip angle pattern as in the additive angle implementation, and with 100 CG iterations. Small-tip-angle design was initialized with zero pulses, and additive angle design was initialized with the small-tip-designed pulses, scaled by 0.75, except in Simulation II, where the scaling factor is varied. This scaling factor corresponds to a nominal initial flip angle of $3 \pi / 8$ radians in the case of $\pi / 2$-pulse design, and $3 \pi / 4$ radians in $\pi$-pulse design. Pulses were designed with a small Tikhonov regularization parameter $\beta=10^{-1}$, except in Simulation II, where $\beta$ was varied. All pulse designs and simulations were performed in MATLAB R2006a (Mathworks, Natick, MA), on a 3.4 GHz Pentium IV workstation with 2 GB RAM.

\section{Numerical Simulations}

Simulations of parallel excitation were carried out assuming an 8 element active rung transmit array (15). Transmit sensitivity patterns were obtained via Finite-Difference Time-Domain simulation (16) of the array at 3.0 Tesla, using a phantom modeled as a $22 \mathrm{~cm}$ diameter lossy cylinder with $\sigma=0.3 \mathrm{~S} / \mathrm{m}$ and $\epsilon_{r}=80$ (Fig. 1a). The ROI for pulse design and error calculation was the phantom interior. The desired pattern, shown in Fig. 1b, was a rectangular block that was centered in the phantom, with dimensions $10 \mathrm{~cm} \times 5 \mathrm{~cm}$.

We designed pulses using spiral and echo-planar (EP) excitation k-space trajectories. Spiral trajectories were single-shot spiral-out with the parameters: maximum amplitude $=4 \mathrm{G} / \mathrm{cm}$, maximum slew rate $=18 \mathrm{G} / \mathrm{cm} / \mathrm{ms}$. Acceleration was achieved via undersampling in the radial direction, resulting in reduction of the excitation field of view (XFOV) of the individual coils' excitation patterns. EP trajectories were designed using the parameters: maximum amplitude $=4 \mathrm{G} / \mathrm{cm}$, maximum slew rate $=15 \mathrm{G} / \mathrm{cm} / \mathrm{ms}$. For these trajectories, acceleration was achieved via undersampling in the blipped (phase-encoded) dimension. We define "speedup factor" as the ratio of the FOV of the desired pattern to the reduced XFOV of the excitation k-space trajectories. The spatial resolution for all trajectories was $0.75 \mathrm{~cm}$, which is twice that of the grid on which the desired patterns were specified. We find that specifying the desired pattern on a grid of finer resolution than the kspace trajectory improves accuracy of both small-tip-angle and additive angle-designed pulses. The sampling period for all pulses was $4 \mu \mathrm{s}$.

\section{Simulation I: Speedup Factor}

In the first simulation, we designed $\frac{\pi}{2}$ - and $\pi$-excitation pulses with a range of speedup factors from 2.4 to 6 , corresponding to an XFOV range from $10 \mathrm{~cm}$ down to $4 \mathrm{~cm}$. We compared performance of the additive angle method to that
Transmit sensitivity patterns for simulations

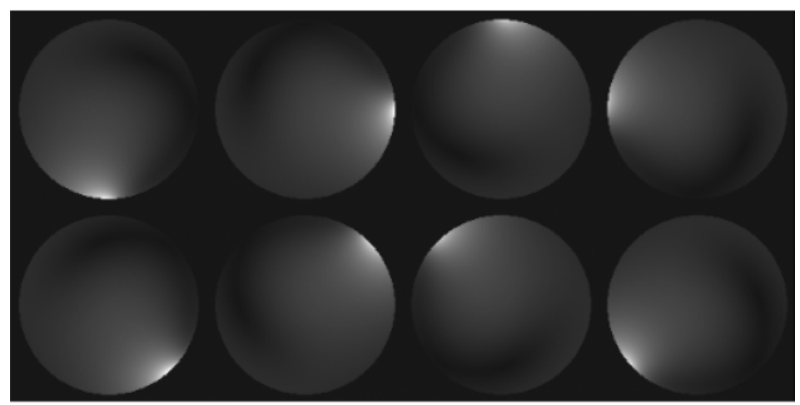

a

Desired pattern for simulations

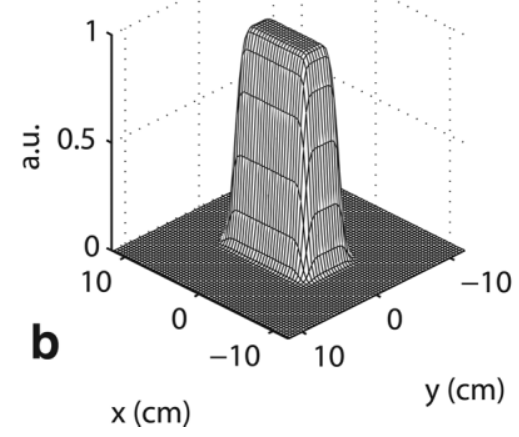

FIG. 1. (a) Magnitude of transmit sensitivity patterns used in simulations of eight-channel parallel excitation. (b) Desired flip angle pattern used in simulations, which was a smoothed $10 \mathrm{~cm} \times 5 \mathrm{~cm}$ rectangular block, whose peak was scaled to $\pi$ and $\frac{\pi}{2}$ for pulse design.

of small-tip-angle design in terms of RMSE between excited and desired flip angle patterns, peak total RF field magnitude, and visual quality. Compute time was also recorded for the additive angle method.

\section{Simulation II: Convergence}

In this simulation we investigated convergence properties of the additive angle method. We repeated additive angle designs of EP (speedup factor $=3$ ) and spiral (speedup factor $=4.8) \pi$-pulses, and varied design parameters that may influence final excitation error. We first varied the nominal flip angle of the initial, small-tip-designed pulse that was used to seed the additive angle method, between 0 and 2, corresponding to seed pulses with nominal flip angles between 0 and $2 \pi$ radians. We then performed designs in which we varied the maximum tip angle of $d^{\text {new }}(\mathbf{x})$ from $\pi / 8$ to $\pi$ radians, with a zero initial seed pulse. Finally, we performed designs over a range of $\beta$-values, from $\log _{10} \beta=-1$ to $\log _{10} \beta=2.5$.

\section{Simulation III: Refocusing}

In this simulation we verified that a $\pi$-pulse designed using the additive angle method will refocus a population of spins possessing a range of off-resonant frequencies, and compared refocusing performance to that of a small-tip designed pulse. EP pulses with speedup 
factor $=3(\mathrm{XFOV}=8 \mathrm{~cm}$, pulse length $3.34 \mathrm{~ms})$ were used in these simulations. We performed 40 simulations that were initialized with magnetization in the transverse plane everywhere in the object, but over a phase range of $0-2 \pi$ radians, in 40 equally spaced angular steps. The initial phase represents the phase accrued due to off-resonance between excitation and TE/2. After application of the pulse, the initial phase was again added to the magnetization's phase, representing the phase accrued between the refocusing pulse (TE/2) and TE. We then summed the magnetization over the phase range, to obtain the net magnetization at TE. Phase evolution due to off-resonance during pulse application was neglected, due to the short duration of the pulse.

\section{Scanner Experiment}

Scanner experiments were performed on a GE 3T Signa Excite Scanner (GE Healthcare, Milwaukee, WI). Because of a lack of parallel transmit hardware, we carried out a single-channel transmit experiment to validate the additive angle design method and compared it to small-tip-angle pulse design. We computed 2D spatially selective inversion pulses that were applied in the spin preparation stage of a spiral GRE sequence. The sequence began with the inversion pulses, followed directly by gradient crushers, which were subsequently followed by standard sinc excitation and a multi-shot spiral-in readout. Ideally, the inversion pulses flip image phase within the inverted region, but leave image magnitude unperturbed.

All transmission and reception was performed using a standard birdcage head coil. A spherical MnCl phantom was used, which expressed a nonuniform transmit field magnitude $(\mathrm{B} 1+)$ in the birdcage coil. The $\mathrm{B} 1+$ field was mapped for one slice in the phantom by acquiring a set of GRE images collected with nominal flip angles $\left(30^{\circ}, 60^{\circ}, 90^{\circ}\right)$, and processing the resulting images with a regularized $\mathrm{B} 1+$ mapping method (17). The imaging parameters for B1+ mapping were as follows: slice thickness = $4.0 \mathrm{~mm} ; \mathrm{FOV}=24 \mathrm{~cm}$; reconstructed matrix size $=64 \times 64$; repetition time $(\mathrm{TR})=8 \mathrm{~s}$; echo time $(\mathrm{TE})=10 \mathrm{~ms}$. The measured B1+ pattern was used to design EP pulses that inverted the $9 \times 3 \mathrm{~cm}$ square desired pattern shown in Fig. 9a. Placement of the inverted region was chosen such that a large B1+ magnitude gradient existed across the long dimension of the region. The EP trajectory was designed with XFOV $=18 \mathrm{~cm}$, spatial resolution = $0.75 \mathrm{~cm}$, maximum slew rate $=15 \mathrm{G} / \mathrm{cm} / \mathrm{ms}$, and sampling period $=4 \mu \mathrm{s}$. To reduce $\mathrm{RF}$ magnitude near the center of k-space, the central 3 phase encodes were designed with a maximum gradient amplitude of $0.5 \mathrm{G} / \mathrm{cm}$, while the outer 22 phase encodes were designed with maximum amplitude $=4 \mathrm{G} / \mathrm{cm}$. These parameters yielded a pulse length of $8.7 \mathrm{~ms}$. Pulse design time, including B1+ map estimation, was recorded. Imaging parameters for the inversion-preparation sequence were as follows: slice thickness $=4.0 \mathrm{~mm}$; FOV $=24 \mathrm{~cm}$; reconstructed matrix size $=128 \times 128 ; \mathrm{TR}=8 \mathrm{~s}$; TE $=20 \mathrm{~ms}$; nominal flip angle (sinc pulse) $=10^{\circ} ; 8$ averages. Excitation gradient waveforms were shifted forward by $150 \mu \mathrm{s}$ to compensate for the delay between RF and gradient channels. Four spiral interleaves were used in both the B1+ mapping and inversion-preparation sequences to reduce readout time so that off-resonance effects during acquisition were minimized. We also acquired images with the inversion pulse magnitude set to zero. These were used to remove the effects of non-uniform transmit and receive sensitivities during sinc excitation and readout by dividing them from of images acquired with the inversion pulses switched on. Images were reconstructed using a fast implementation of the off-resonance compensated conjugate phase method (18). It used field maps estimated from two images acquired with a TE difference of $2.5 \mathrm{~ms}$ (19).

\section{RESULTS}

\section{Simulation I: Acceleration}

Figure 2 shows simulated $\pi$-excitation patterns. In Fig. 2a,c, the small-tip-designed pulses produce significant erroneous excitation outside the inverted blocks. In the EP case, we see the well-known bandwidth narrowing effect (20) that occurs when small-tip-angle Cartesian pulses are scaled to large tip-angles. This effect manifests as a spatially narrow inversion region, compared to the wider region the pulses would correctly excite if scaled to produce small tip-angles. For both EP and spiral trajectories, excitation accuracy is dramatically improved by the additive angle method. In Fig. 3a,b, we see that inversion pulses designed using the additive angle method achieve lower RMSE over the full speedup factor range, without a significant increase in peak RF magnitude. Improvement in accuracy is most dramatic for the EP trajectory.
Small-Tip, EP

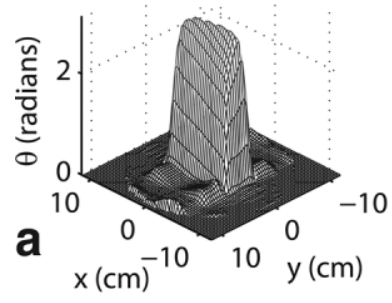

Small-Tip, Spiral

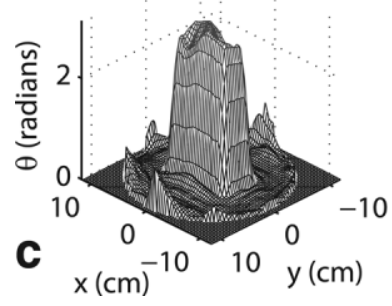

Additive Angle, EP

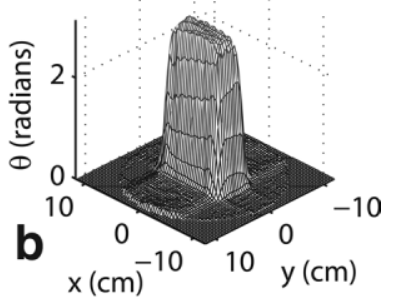

Additive Angle, Spiral

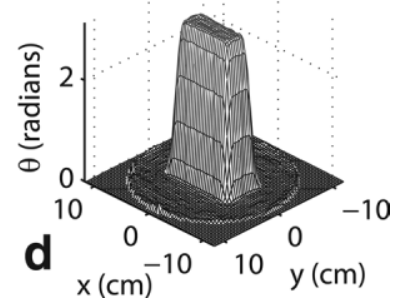

FIG. 2. Simulated $\pi$ excitation patterns produced by pulses designed with the small-tip $(\mathbf{a}, \mathbf{c})$ and additive angle $(\mathbf{b}, \mathbf{d})$ methods, for an EP trajectory $(a, b)$ with speedup factor $=3(X F O V=8 \mathrm{~cm}$, pulse length $3.34 \mathrm{~ms}$ ), and a spiral trajectory (c,d) with speedup factor $=4.8$ (XFOV $=5 \mathrm{~cm}$, pulse length $1.16 \mathrm{~ms}$ ). For both trajectories, the additive angle method yields pulses that produce patterns with reduced erroneous excitation outside the block, and improved uniformity inside the block. 

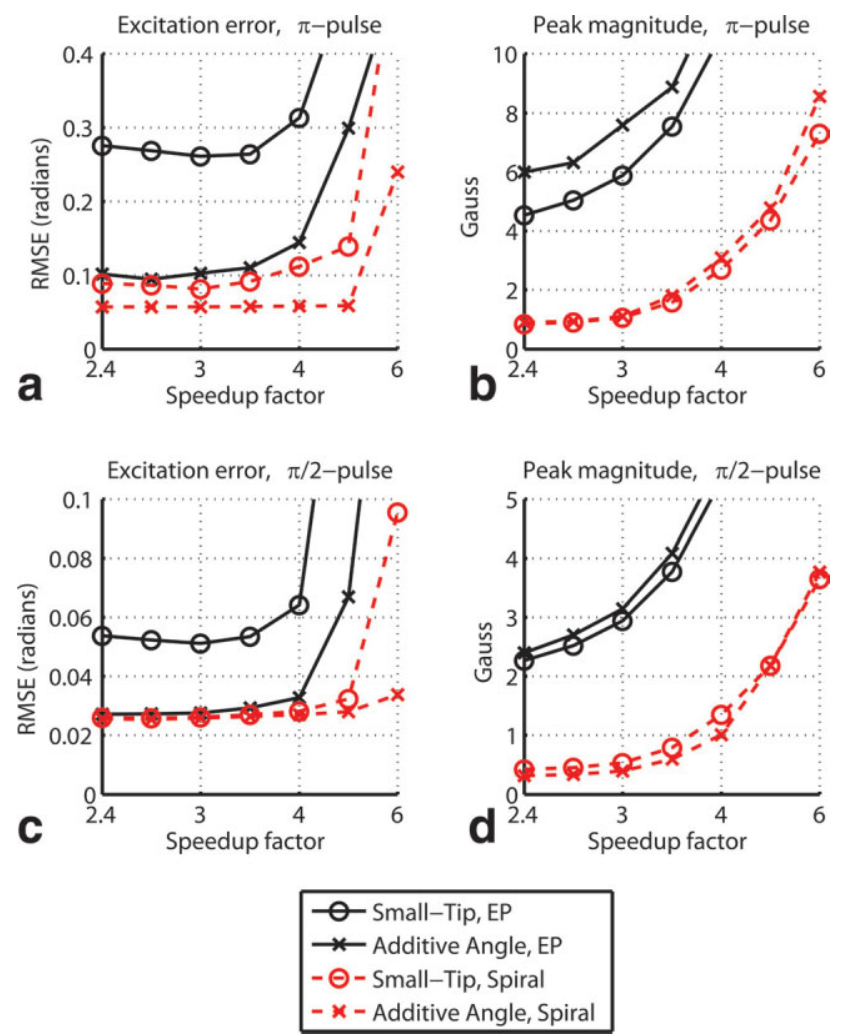

FIG. 3. Comparison of excitation accuracy and peak RF magnitude between pulses designed using the small-tip and additive angle methods, over a range of speedup factors. For both $\pi$ - and $\frac{\pi}{2}$-pulses $(\mathbf{a}, \mathbf{b})$, the additive angle method achieves lower error, without a significant increase in pulse power. Improvement in accuracy with the additive angle method is most significant for EP and spiral $\pi$-pulses, and for EP $\frac{\pi}{2}$-pulses.

Figure 4 plots coil 1's pulse magnitude for the $\pi$-excitation pulses of Fig. 2. We observe that in the EP case (Fig. 4a), large changes in pulse shape were required to achieve high excitation accuracy, while in the spiral case (Fig. 4b), smaller perturbations resulted in significantly improved accuracy. Figure 5 shows simulated $\frac{\pi}{2}$-excitation profiles, and we again see an improvement in excitation accuracy for the additive angle case. As shown in Fig. 3c,d, this improvement is maintained over the simulated speedup factor range, particularly in the EP case, again without a significant increase in peak RF magnitude. Additionally, for both trajectories, the phase within the blocks excited by additive angle pulses is highly uniform, as shown in Fig. 5e,f. In the EP case of Fig. 5b,e, an average (RMS) phase deviation of $4.8^{\circ}$ was measured in the block, while in the spiral case of Fig. 5d,f, an RMS deviation of $3.5^{\circ}$ was measured. This is a welcome result, considering that the excited magnetization's phase is controlled only implicitly via the desired pattern used in the initial small-tip-angle pulse design.

Figure 6 plots design times required for convergence. Spiral design times are shorter than EP design times for both $\pi$ - and $\frac{\pi}{2}$-pulses, with the exception of the highest speedup factors for which accurate EP pulses are not reached. Longer EP design time is most likely due to the longer duration of the EP trajectory for a given speedup factor, and the higher level of error produced by the small-tip-designed pulses used to seed EP pulse design. Compared to spiral design times, the longer EP times are also in agreement with the larger changes in waveform shape created by our method, shown in Fig. 4. Design time for $\pi$-excitation pulses is generally longer than that for $\frac{\pi}{2}$-pulses in both EP and spiral pulse design, which can be attributed to the higher level of error produced by the small-tip-designed $\pi$-pulses used to seed our method. Bloch simulation/small-excitation pulse design iterations required for convergence were roughly proportional to design time. EP $\pi$-pulse design required between 10 and 20 iterations, while spiral $\pi$-pulse design required between 7 and 16 iterations. For $\frac{\pi}{2}$-pulse design, the EP trajectory required between 7 and 12 iterations, while the spiral trajectory required between 5 and 15 iterations. In comparison to these measured times, design times for exact Bloch-equation based methods are generally an order of magnitude longer.

\section{Simulation II: Convergence}

Figure 7 shows results of our convergence investigation. From the designs in which we varied the nominal flip angle of the initial small-tip-designed pulse (Fig. 7a), we see that the additive angle method converges to the same error level over the range of nominal flip angles from 0 to $\pi$ radians. In fact, the method continues to converge to the same error even for larger nominal flip angles that would not be used in practice. Figure $7 \mathrm{~b}$ shows that the method is also robust to the maximum magnitude of $\mathbf{d}_{\text {new }}(\mathbf{x})$. Figure 7c shows that the final RMS error of the pulses is a smooth, increasing
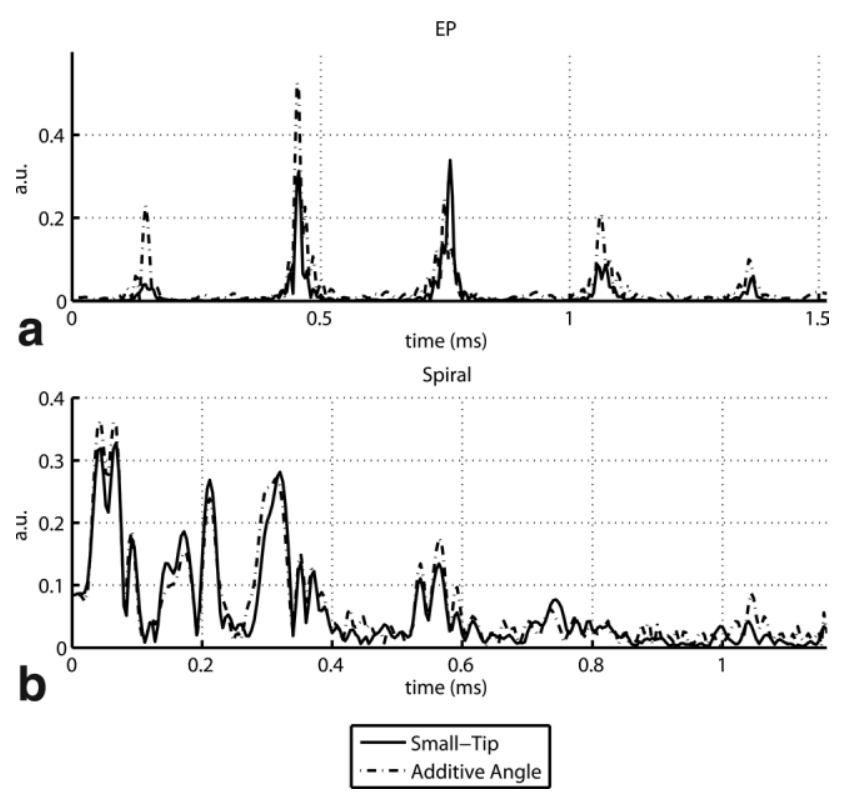

FIG. 4. Magnitude of the $\pi$-excitation pulses of Fig. 2 for coil 1 . (a) In the EP case (pulse plotted for the central five phase encode lines only), large changes in pulse shape resulted in significantly decreased RMS error, from 0.26 radians for the small-tip designed pulses, to 0.10 radians for the additive angle-designed pulses. (b) For spiral pulses, smaller perturbations decreased RMS error from 0.14 radians to 0.06 radians. 
Small-Tip, EP

a

Small-Tip, Spiral
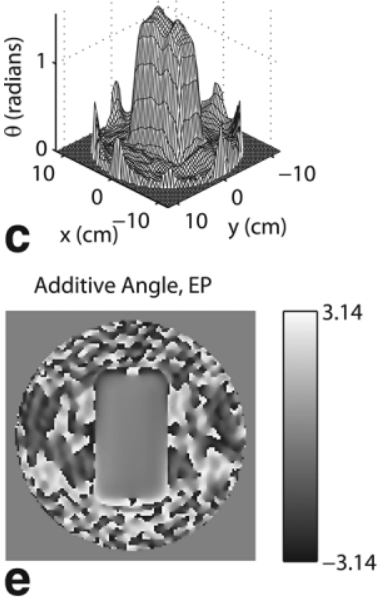

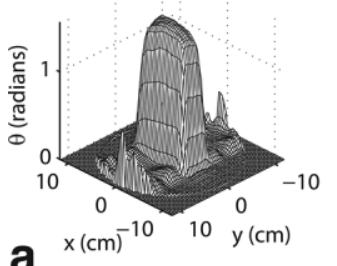

Additive_Anqle, EP
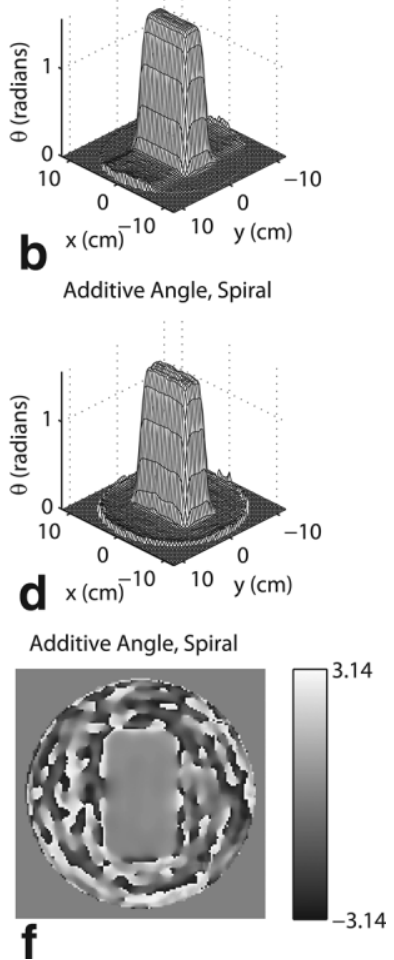

FIG. 5. Simulated $\frac{\pi}{2}$ excitation patterns produced by pulses designed with the small-tip (a,c) and additive angle (b,d) methods. For both the EP trajectory $(a, b)$ with speedup factor $=4(X F O V=6 \mathrm{~cm}$, pulse length $2.7 \mathrm{~ms}$ ), and the spiral trajectory $(\mathrm{c}, \mathrm{d})$ with speedup factor $=6$ (XFOV $=4 \mathrm{~cm}$, pulse length $1 \mathrm{~ms})$, pulses designed with the additive angle method produce patterns with reduced erroneous excitation outside the block, and improved uniformity inside the block. (e,f) Pulses designed using the additive angle method excite magnetization with uniform phase, even though phase is allowed to drift during the design process.

function of the Tikhonov regularization parameter $\beta$. Using a higher $\beta$ results in a higher RMS error, but reduces peak RF magnitude, as shown in Fig. 7, and can be tuned to balance the two metrics. EP and spiral pulse designs possess similar sensitivity to all design parameters.

\section{Simulation III: Refocusing}

Figure 8 shows simulation results of small-tip and additive angle-designed $\pi$-pulses used for refocusing. In Fig. 8a, the magnetization magnitude subsequent to application of the small-tip-designed $\pi$-pulse of Fig. 2a is highly non-uniform within the refocused block, and exhibits a narrowing effect similar to that of the pulse's flip angle pattern. Some magnetization is also refocused outside the block. Figure $8 \mathrm{~b}$ shows the magnetization phase at TE, which is relatively uniform within the block, with an RMS deviation of $9^{\circ}$. In contrast, Fig. 8c shows that the magnetization after application of the additive angle-designed pulse is uniform inside the block, and no magnetization is refocused outside it. In Fig. 8d, we see that for the additive angle pulse, magnetization phase is spatially varying

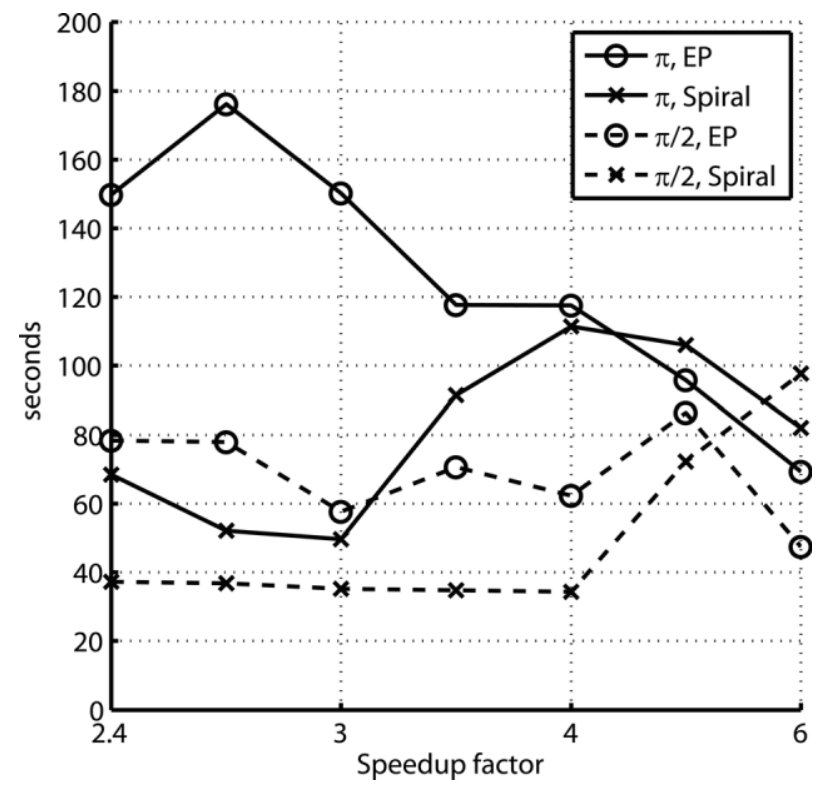

FIG. 6. Measured design times for simulations. The design times for $\frac{\pi}{2}$-pulses are shorter than those for $\pi$-pulses. Additionally, design times for spiral pulses are generally shorter than those for EP pulses. This is most likely due to the shorter length of spiral pulses compared to EP pulses, and the lower error achieved by the small-tip-designed spiral pulses used to seed the additive angle method, compared to EP seed pulses.
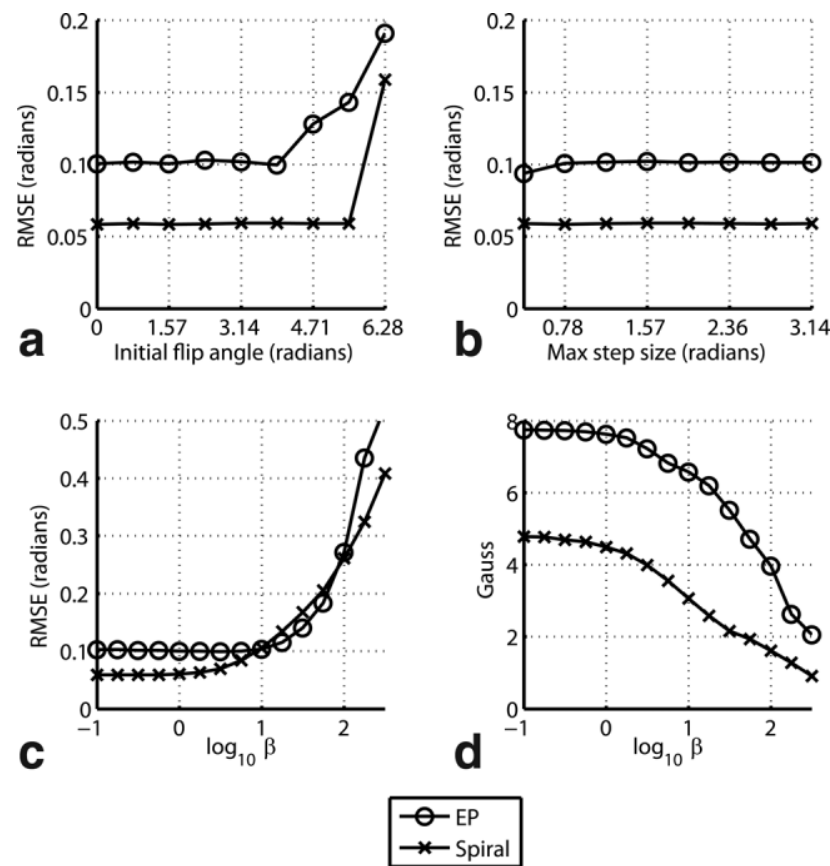

FIG. 7. Influence of design parameters on convergence and peak RF magnitude. Additive angle designs of EP (speedup factor $=3$ ) and spiral (speedup factor $=4.8) \pi$-pulses were repeated over a range of design parameters, to investigate the method's convergence properties. $(\mathbf{a}, \mathbf{b})$ The additive angle method is robust to both the nominal flip angle of the small-tip-designed pulse used to seed the method, and the maximum flip angle allowed in $d^{\text {new }}(x)$. (c,d) The Tikhonov regularization parameter $\beta$ can be increased to mitigate peak RF magnitude, at the cost of increased excitation error. 

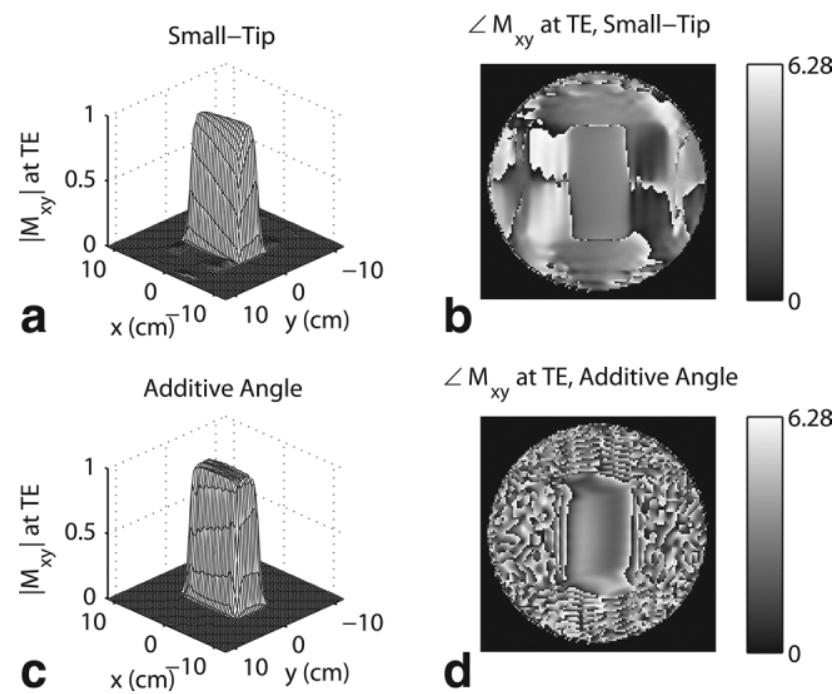

FIG. 8. Comparison of small-tip and additive angle-designed EP $\pi$-pulses applied as refocusing pulses. In this simulation, the pulses of Fig. 2a,b were used to excite magnetization initially in the transverse plane, and over a range of transverse-plane phase angles. (a) The small-tip-designed pulse fails to uniformly rotate magnetization by $\pi$ radians within the block, resulting in an inhomogeneous magnetization pattern at TE. Furthermore, the small-tip-designed pulse partially refocuses magnetization outside the block. (b) In comparison, the additive angle-designed pulse fully refocuses magnetization within the block, and does not refocus magnetization outside the block. (b,d) The net phase of magnetization at TE is spatially varying for both pulses, though the additive-angle pulse leaves more phase variation within the refocused block.

within the block at TE, with a higher RMS phase deviation of $34^{\circ}$.

\section{Scanner Experiment}

Figure $9 \mathrm{~b}$ shows a magnitude profile of the estimated B1+ map, which expresses significant inhomogeneity over the phantom. Within the desired inversion pattern, the B1+ map has a maximum magnitude of 0.74 Gauss/full RF magnitude, a minimum of 0.51 Gauss/full RF magnitude, and a mean of 0.66 Gauss/full RF magnitude. A pulse designed without B1+ compensation and tuned to excite $\pi$ radians at the mean B1+ magnitude would produce flip angles between 2.43 and 3.5 radians over the excited region. Figure 9c,d show images obtained using the inversion-preparation sequence. The effects of nonuniform B1+ during sinc excitation and B1- during signal reception have been removed from these images using reference images obtained with the inversion pulses set to zero. As in our simulations, we see that the small-tip-designed pulses suffer from a bandwidth-narrowing effect (20) in the phase-encode dimension. The effect is manifested as the narrowed $M_{y}$ profile in Fig. 9e, and the non-uniform image intensity of the inverted region of Fig. 9c. In contrast, the additive angle-designed pulse inverts an accurate rectangular region (Fig. 9d), in which the image intensity is more uniform (Fig. 9d,e). The intensity inside the inverted region is close to that outside it, indicating that a full inversion was achieved in the square. Pulse design time, including B1+ map image reconstruction and estimation, was $46.3 \mathrm{sec}$.

\section{DISCUSSION AND CONCLUSIONS}

We have presented a new method for fast large-tip-angle $\mathrm{RF}$ pulse design in multidimensional parallel excitation, and have shown that it may be used to design $\pi$ - and $\frac{\pi}{2}$ excitation pulses. We have demonstrated that these pulses produce flip angle patterns with significantly improved accuracy, compared to pulses designed using small-tipangle methods. In the case of spiral trajectories, improvement in accuracy is most dramatic at high speedup factors, corresponding to large reductions in pulse length. This is
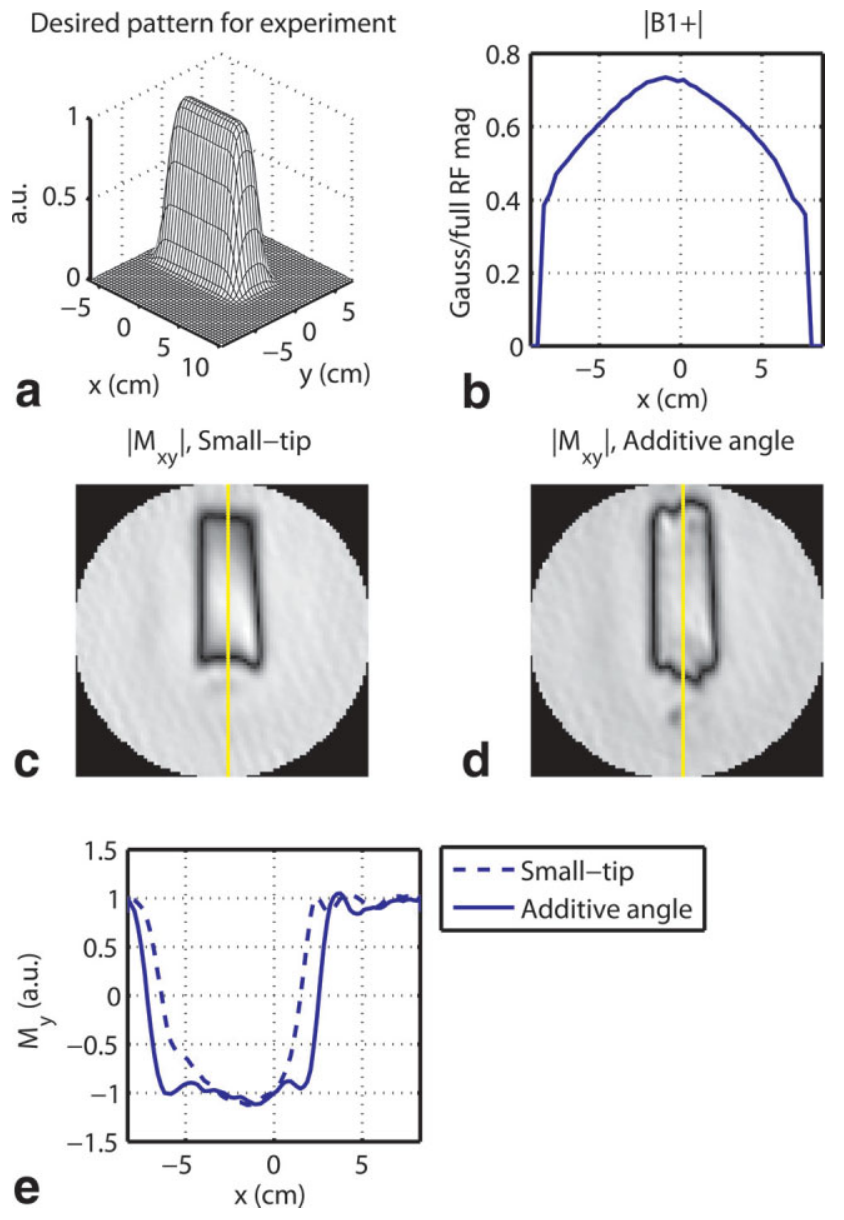

FIG. 9. Experimental results. (a) The desired flip angle pattern was a smoothed $9 \times 3 \mathrm{~cm}$ square, whose peak was scaled to $\pi$ for inversion pulse design. (b) Magnitude profile through the center of the object's B1+ field map, along the dashed lines in (c,d). (c,d) Magnitude images of the phantom after application of the small-tip-designed pulse (c) and additive angle-designed pulse (d). (e) $M_{y}$ profiles taken through the center of the phantom, as indicated by the dashed lines in $(\mathrm{c}, \mathrm{d})$. The small-tip-designed pulse produces a pattern that is narrowed in the phase-encode direction, while the additive angle pulse produces an accurate square inversion region that matches the desired pattern. The $M_{y}$ profiles verify that magnetization phase in the inverted region is shifted by $\pi$ relative to magnetization outside. [Color figure can be viewed in the online issue, which is available at www.interscience.wiley.com.] 
due to the fact that pulses designed with spiral trajectories satisfy the linear-class conditions (6) at low speedup factors, and may thus be scaled to produce accurate large-tipangle excitation patterns. At high speedup factors however, increased pulse magnitude violates the small-RF approximation used to derive the linear class (4), and a nonlinear design method such as the one presented here must be used. In the case of EP trajectories, we showed that a significant improvement in accuracy is achieved over the full range of speedup factors. The EP trajectory does not satisfy the linear-class conditions, and so a non-linear method must be used at all speedup factors. We showed experimentally that our method is also capable of designing accurate large-tip-angle single-channel EP pulses. Though not presented here, we have also designed 'B1+ shimming' pulses using three-dimensional fast $k_{z}$ trajectories $(21,22)$, and have found that the additive angle method produces accurate three-dimensional large-tip-angle pulses in that scenario as well.

Our method presents two opportunities to further reduce compute time by parallelizing computations. First, all small-tip-angle pulse designs require the evaluation of as many NUFFT's as there are coils, at each iteration of the CG algorithm. Because the NUFFT's are independent of each other, they can be computed in parallel to achieve a reduction in small-tip-angle design times. The second opportunity is in Bloch simulation, which may be parallelized by dividing the simulation spatially or temporally. Although our method already produces accurate pulses in a short time, these improvements would make online large-tip-angle parallel pulse design even more practical.

Many of the pulses we designed in simulation possessed high peak RF magnitudes and could not be feasibly deployed on human MR scanners. We showed in simulations that one approach to obtaining usable pulses is to increase Tikhonov regularization, which reduces peak $\mathrm{RF}$ magnitude at the cost of increased excitation error. Another approach is to modify the k-space trajectories using variable-XFOV $(23,24)$ or variable-rate strategies $(25$, 26 ) to increase sampling density in regions of k-space where the pulses are expected to possess high magnitude. Both strategies will reduce $\mathrm{RF}$ magnitude at the cost of increased pulse length. In the design of large-tip-angle pulses, the k-space regions of high RF magnitude can be determined either from prior pulse designs, or by intuition from the small-tip-angle pulse design scenario.

In our method, the excited transverse magnetization's phase is controlled only initially via the desired pattern for small-tip-angle pulse design, and is allowed to vary both spatially and between design iterations. Deviations from the initial phase are introduced by Bloch equation nonlinearity. In our simulations we found that phase deviation within the $\frac{\pi}{2}$-excitation patterns was small, suggesting that a multidimensional pulse designed using our method could still be used to perform slice-selection in one dimension, with negligible signal loss due to signal cancellation through the slice. We also demonstrated that $\pi$-excitation pulses may be employed for refocusing, though there are larger variations in the resulting phase in this case. While this is not problematic for scenarios in which the pulses are applied in the imaging plane, it may reduce their utility for cases where signal is averaged across the refocused region.

In conclusion, we have introduced a method for large-tipangle parallel RF pulse design that is capable of producing accurate multidimensional pulses in a short time. The method is simple to implement, provided one already has the software tools available to design small-tip-angle parallel excitation pulses. Through spiral and EP pulse design simulations, we demonstrated that the method produces pulses of high accuracy that may be used for excitation, inversion, and refocusing.

\section{SYMBOLS}

- $\theta(\mathbf{x})$ : flip angle pattern

- x: spatial location (vector)

- k: excitation k-space trajectory waveforms (vectors, continuous functions of time)

- $s_{r}(\mathbf{x})$ : coil $r$ 's transmit sensitivity (complex, continuous function of space)

- $t$ : time

- $b_{r, j}$ : samples of RF pulses (complex)

- $N_{t}$ : number of time points in the RF pulses

- $R$ : number of coils

- $\Delta t$ : sampling period of pulses

- $\gamma$ : gyromagnetic ratio

- $N_{s}$ : number of spatial points

- $r$ : coil index

- $i$ : imaginary number, also spatial index

- $\angle M_{x y}(\mathbf{x})$ : phase of transverse magnetization $M_{x y}(\mathbf{x})$

- $\Psi$ : cost function for designing correction pulses

- $\tilde{\mathbf{b}}_{r}$ : vector of correction RF pulse samples for coil $r$

- $\mathbf{S}_{r}$ : diagonal matrix of sensitivity values for coil $r$

- A: Fourier system matrix

- $\mathbf{d}^{\text {new }}$ : vector of samples of desired pattern for designing correction pulses

- W: diagonal matrix specifying Region of Interest (ROI) for pulse design

- $\beta$ : Tikhonov regularization parameter

- $\mathbf{b}_{r}$ : vector of initial RF pulse samples for coil $r$

\section{REFERENCES}

1. Katscher U, Börnert P, Leussler C, van den Brink JS. Transmit SENSE. Magn Reson Med 2003;49:144-150.

2. Zhu Y. Parallel excitation with an array of transmit coils. Magn Reson Med 2004;51:775-784.

3. Griswold MA, Kannengiesser S, Muller M, Jakob PM. Autocalibrated accelerated parallel excitation (Transmit-GRAPPA). In Proceedings 13th Scientific Meeting, International Society for Magnetic Resonance in Medicine, Miami Beach; 2005, p 2435.

4. Grissom WA, Yip CY, Zhang Z, Stenger VA, Fessler JA, Noll DC. Spatial domain method for the design of RF pulses in multicoil parallel excitation. Magn Reson Med 2006;56:620-9.

5. Pauly JM, Nishimura DG, Macovski A. A k-space analysis of small-tipangle excitation. Magn Reson Med 1989;81:43-56.

6. Pauly JM, Nishimura DG, Macovski A. A linear class of large-tip-angle selective excitation pulses. J Magn Reson 1989;82:571-587.

7. $\mathrm{Xu} \mathrm{D,} \mathrm{King} \mathrm{KF,} \mathrm{Zhu} \mathrm{Y,} \mathrm{McKinnon} \mathrm{GC,} \mathrm{Liang} \mathrm{ZP.} \mathrm{A} \mathrm{noniterative}$ method to design large-tip-angle multidimensional spatially-selective radio frequency pulses for parallel transmission. Magn Reson Med 2007;58:326-334.

8. Ulloa JL, Callaghan M, Irarrazaval P, Hajnal J, Guarini M. Calculation of B1 pulses for RF shimming at arbitrary flip angle using multiple transmitters. In Proceedings 14th Scientific Meeting, International Society for Magnetic Resonance in Medicine, Seattle; 2006. p 3016. 
9. Setsompop K, Zelinski AC, Alagappan VA, Fontius UJ, Hebrank F, Schmitt F, Wald LL, Adalsteinsson E. High flip angle slice selective parallel RF excitation on an 8-channel system at 3T. In Proceedings 16th Scientific Meeting, International Society for Magnetic Resonance in Medicine, Berlin; 2007. p 677.

10. Xu D, King KF, Zhu Y, McKinnon G, Liang Z-P. Multidimensional arbitrary-flip-angle parallel transmit pulse design using an optimal control approach. In Proceedings 15th Scientific Meeting, International Society for Magnetic Resonance in Medicine, Berlin; 2007. p 1696.

11. Yip CY, Fessler JA, Noll DC. Advanced three-dimensional tailored $\mathrm{RF}$ pulse for signal recovery in $\mathrm{T} 2{ }^{*}$-weighted functional magnetic resonance imaging. Magn Reson Med 2006;56:1050-9.

12. Lebsack ET, Wright SM. Iterative RF pulse refinement for magnetic resonance imaging. IEEE Trans Biomed Eng 2002;49:41-48.

13. Ngo JT, Morris PG. General solution to the NMR excitation problem for noninteracting spins. Magn Reson Med 1987;5:217-237.

14. Fessler JA, Sutton BP. Nonuniform fast fourier transforms using minmax interpolation. Signal Proces, IEEE Trans [Acoustics, Speech, Signal Process, IEEE Trans], 2003;51:560-574.

15. Kurpad KN, Boskamp EB, Wright SM. A parallel transmit volume coil with independent control of currents on the array elements. In Proceedings 13th Scientific Meeting, International Society for Magnetic Resonance in Medicine, Miami Beach; 2005. p 16.

16. Wright SM. 2D full-wave modeling of SENSE coil geometry factors at high fields. In Proceedings 10th Scientific Meeting, International Society for Magnetic Resonance in Medicine, Honolulu; 2002. Vol 10; p 854.
17. Funai A, Fessler JA, Grissom W, Noll DC. Regularized B1+ map estimation in MRI. In Biomedical Imaging: Macro to Nano, 2007. 4th IEEE International Symposium on Biomedical Imaging; 2007.

18. Noll DC, Meyer CH, Pauly JM, Nishimura DG, Macovski A. A homogeneity correction method for magnetic resonance imaging with timevarying gradients. IEEE Trans Med Imag 1991;10:629-637.

19. Schneider E, Glover G. Rapid in vivo proton shimming. Magn Reson Med 1991;18:335-347.

20. Pauly JM, Spielman D, Macovski A. Echo-planar spin-echo and inversion pulses. Magn Reson Med 1993;29:776-782.

21. Saekho S, Yip CY, Noll DC, Boada FE, Stenger VA. Fast-kz threedimensional tailored radiofrequency pulse for reduced B1 inhomogeneity. Magn Reson Med 2006;55:719-724.

22. Zhang Z, Yip CY, Grissom W, Noll DC, Boada FE, Stenger VA. Reduction of transmitter B1 inhomogeneity with transmit SENSE slice-select pulses. Magn Reson Med 2007;57:842-847.

23. Christoph Schroder, Peter Bornert, Bernd Aldefeld. Spatial excitation using variable-density spiral trajectories. J Magn Reson Imag 2003;18:136-141.

24. Stenger VA, Boada FE, Noll DC. Variable-density spiral 3D tailored RF pulses. Magn Reson Med 2003;50:1100-1106.

25. Conolly S, Nishimura DG, Macovski A, Glover G. Variable-rate selective excitation. J Magn Reson 1988;78:440-458.

26. Graesslin I, Biederer S, Schweser F, Zimmerman K-H, Katscher U, Börnert P. SAR reduction for parallel transmission using VERSE and k-space filtering. In Proceedings 16th Scientific Meeting, International Society for Magnetic Resonance in Medicine, Berlin; 2007. p 674. 